\title{
Equilibrium Configurations of the Noncircular Cross-Section Elastic Rod Model with the Elliptic KB Method
}

\author{
Yongzhao Wang, ${ }^{1,2}$ Qichang Zhang, ${ }^{1}$ and Wei Wang ${ }^{1}$ \\ ${ }^{1}$ Tianjin Key Laboratory of Nonlinear Dynamics and Chaos Control, School of Mechanical Engineering, \\ Tianjin University, Tianjin 300072, China \\ ${ }^{2}$ School of Mathematics and Statistics, Xuchang University, Xuchang 461000, China \\ Correspondence should be addressed to Qichang Zhang; qzhang@tju.edu.cn
}

Received 3 November 2014; Accepted 21 February 2015

Academic Editor: Lakshmanan Shanmugam

Copyright (c) 2015 Yongzhao Wang et al. This is an open access article distributed under the Creative Commons Attribution License, which permits unrestricted use, distribution, and reproduction in any medium, provided the original work is properly cited.

\begin{abstract}
The mechanical deformation of DNA is very important in many biological processes. In this paper, we consider the reduced Kirchhoff equations of the noncircular cross-section elastic rod characterized by the inequality of the bending rigidities. One family of exact solutions is obtained in terms of rational expressions for classical Jacobi elliptic functions. The present solutions allow the investigation of the dynamical behavior of the system in response to changes in physical parameters that concern asymmetry. The effects of the factor on the DNA conformation are discussed. A qualitative analysis is also conducted to provide valuable insight into the topological configuration of DNA segments.
\end{abstract}

\section{Introduction}

DNA is a long polymer made of millions (or even hundreds of millions) of nucleotides arranged in two complementary strands forming a double helix. Genetic information in living cells is carried in the linear sequence of nucleotides in DNA. Conformational features and mechanical properties of DNA in vivo (such as supercoil formation and bend/twist rigidity) play an important role in its packing, gene expression, protein synthesis [1,2], protein transport [3], and so forth, as misfolding of DNA has become the major cause of many illnesses, such as paroxysmal nocturnal hemoglobinuria (PNH) disease [4]. Thus, it is necessary to understand the basic mechanisms of DNA folding that leads to new ways for preventing such diseases. In recent years, geometrical configuration of a DNA chain has attracted considerable attentions.

The elastic properties of ds-DNA molecules are believed to play an important role in many biological functions [5, 6]. Over the past two decades, the elastic properties have been extensively studied with the development of singlemolecule manipulation techniques [7-11] and experiment capabilities. Analytical models based on classical elasticity theory [12-14] have no spatial/temporal limitations and have widely been used to study the DNA configurations. The elastic rod model to research the flexible structures is to assume that they are made of an elastic material obeying the appropriate laws of elasticity. The well-known Kirchhoff models for rods are widely used to describe the stationary states of elastic filaments within the approximation of linear elasticity theory through a system of six coupled ordinary differential equations [12]. In 1859, Kirchhoff discovered that the equations that describe the thin elastic rod in equilibrium are mathematically identical to those used to describe the dynamics of the heavy top. Shi and Hearst [15] derived a time-independent, one-dimensional nonlinear Schrödinger equation for the stationary state configurations of supercoiled DNA. Xue et al. [16] extended the Schrödinger equation to fit the noncircular Kirchhoff elastic rod by using the complex rigidity. Wang et al. $[17,18]$ rebuilt the initial Kirchhoff equations in a complex style to suit the character of obvious asymmetry and the periodically varying bending coefficients, which is embodied on the cross-section by considering the mathematical background of DNA double helix, and introduced a complex form variable solution of the torque to obtain a simplified second ordinary differential equation 
with single variable. However, in above work, the complex expression of $M_{3}$ according to the complex normal form method is not accurate; we will correct this error in the following section.

In recent years, the analysis of static and dynamic configurations of elastic rod has drawn great attentions. In this paper, we will consider the revised reduced Kirchhoff equations of the noncircular cross-section elastic rod characterized by the inequality of the bending rigidities. It is crucial to find the exact or approximate solutions for the revised simplified second ordinary differential equation in order to investigate the configurations of DNA segments. Reference [19] applied the enhanced cubication method to develop approximate solutions for the most common nonlinear oscillators and leads to amplitude-time response curves and angular frequency values. Reference [20] developed a nonlinear transformation approach to obtain the equivalent representation form of conservative two-degree-of-freedom nonlinear oscillators. Lai and Chow [21] used Jacobi elliptic Krylov-Bogoliubov (KB) method to find two families of exact solutions for oscillators with quadratic damping and mixedparity nonlinearity. Motivated by the above literatures review, this paper focuses on accurate solutions for the reduced Kirchhoff equations and undertakes a qualitative analysis of the topological configuration of DNA segments.

The paper is divided into four parts. In the next section, the reduced form of Kirchhoff's equations is revised. In the third section, the periodic solutions of the equations are found and the effects of anisotropic on configuration of DNA are discussed. Finally, some conclusions are drawn and the paper is closed.

\section{The Reduced Form of Kirchhoff Equations}

As a coarse-grained description, a DNA can be approximately regarded as a thin flexible and inextensible rod or string $[12,15,16]$. The classical theory of elasticity describes the geometry of an elastic rod in terms of its center line $\mathbf{R}=$ $\mathbf{R}(s)=(x(s), y(s), z(s))$, three-dimensional curve parameterized by its arc-length $s$. In presence of external moment $\mathbf{m}$ and external load $\mathbf{f}$ which are distributed along the central axis $\mathbf{R}$ (as show in Figure 1), the static Kirchhoff equations in body fixed frame are as follows:

$$
\begin{gathered}
\frac{d \mathbf{F}}{d s}+\mathbf{f}=0, \\
\frac{d \mathbf{M}}{d s}+\mathbf{e}_{3} \times \mathbf{F}+\mathbf{m}=0,
\end{gathered}
$$

where $\mathbf{F}$ and $\mathbf{M}$ denote the elastic force and moment, respectively.

As shown in $[17,18]$, the complex vector bases $\mathbf{D}_{i}$ are used to substitute the real form vectors $\left\{\mathbf{e}_{1}, \mathbf{e}_{2}, \mathbf{e}_{3}\right\}$ :

$$
\begin{gathered}
\mathbf{D}_{a}=\frac{\mathbf{e}_{1}-i \mathbf{e}_{2}}{2}, \\
\overline{\mathbf{D}_{a}}=\frac{\mathbf{e}_{1}+i \mathbf{e}_{2}}{2}, \\
\mathbf{D}_{3}=\mathbf{e}_{3} .
\end{gathered}
$$

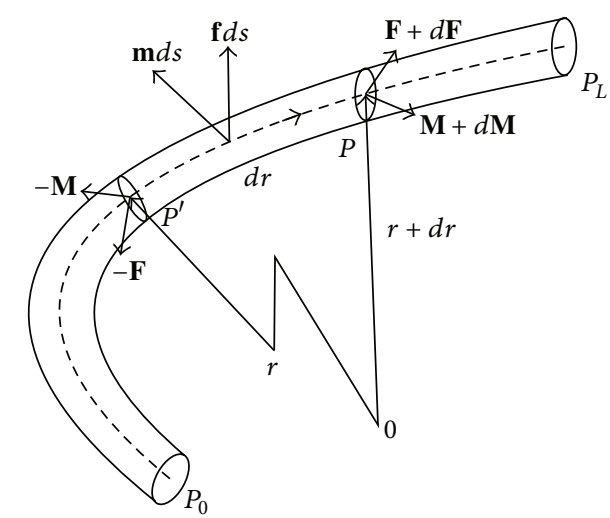

FIGURE 1: The deformed state loaded by forces and moments per unit length [17].

Thus, the complex Kirchhoff equations in the case of zero external moment $\mathbf{m}$ can be written out in terms of $\mathbf{D}_{i}$, such as

$$
\begin{aligned}
F_{a}^{\prime}+i\left(F_{a} \omega_{3}-F_{3} \omega_{a}\right)+f_{a} & =0, \\
M_{a}^{\prime}+i\left(M_{a} \omega_{3}-M_{3} \omega_{a}+F_{a}\right) & =0, \\
F_{3}^{\prime}+\frac{i}{2}\left(\overline{F_{a}} \omega_{a}-F_{3} \overline{\omega_{a}}\right)+f_{3} & =0, \\
M_{3}^{\prime}+\frac{i}{2}\left(\overline{M_{a}} \omega_{a}-M_{a} \overline{\omega_{a}}\right) & =0,
\end{aligned}
$$

where $x_{a}=x_{1}+i x_{2}$ and $\overline{x_{a}}$ denotes the complex conjugate vector of $x_{a}$ with $\left\{x_{a}, \overline{x_{a}}, x_{3}\right\}$ being the projection on each complex axis. $M_{a}=M_{1}+i M_{2}=A \omega_{1}+i B \omega_{2}, M_{3}=C \omega_{3}$, where $M_{1}$ and $M_{2}$ are the bending moments and $M_{3}$ is the twisting component along the rod.

In [17, 18], a complex expression of $M_{3}$ is brought, which is analogous to the complex normal form method. However, this expression is inaccurate; we revised the expression as follows:

$$
M_{3}=\frac{\alpha}{2}\left(M_{a} \overline{\omega_{a}}+\overline{M_{a}} \omega_{a}\right),
$$

where $\alpha$ is per unit length scale. In this case, both sides of (4) are consistent for the dimension, and it is easy to find a new way to improve the reduced form while $\alpha$ is a constant or a function of arc-length.

That produces the expressions of $\omega_{1}$ and $\omega_{2}$ by solving (3) and (4):

$$
\begin{aligned}
& \omega_{1}=\frac{(A-B) M_{3} \pm \sqrt{(A-B)^{2} M_{3}^{2}-4 A B \alpha^{2}\left(M_{3}^{\prime}\right)^{2}}}{2 A \alpha M_{3}^{\prime}} \omega_{2}, \\
& \omega_{2}=\sqrt{\frac{(A-B) M_{3} \mp \sqrt{(A-B)^{2} M_{3}^{2}-4 A B \alpha^{2}\left(M_{3}^{\prime}\right)^{2}}}{2 \alpha(A-B) B}} .
\end{aligned}
$$


Following with [17], the reduced form of Kirchhoff equations can be expressed as

$$
\begin{aligned}
& \frac{d^{2} M_{3}}{d s^{2}}-\frac{(A-B)^{2}+8 \alpha^{2}(A+B) H}{8 \alpha^{2} A B} M_{3} \\
& +\frac{(A-B)^{2}+(A+B) C}{2 \alpha A B C} M_{3}{ }^{2} \\
& -\frac{4 A B-3(A+B) C}{2 A B C^{2}} M_{3}{ }^{3}+\frac{2 \alpha}{C}\left(\frac{d M_{3}}{d s}\right)^{2}=0,
\end{aligned}
$$

where $H$ is the Hamiltonian of system.

We simplify (6) by first making all the variables dimensionless, that is, defining

$$
\begin{gathered}
\bar{s}=\frac{s}{\alpha}, \\
\overline{M_{3}}=\frac{M_{3}}{E I}, \\
\bar{A}=\frac{A}{E I}, \\
\bar{B}=\frac{B}{E I}, \\
\bar{C}=\frac{C}{E I}, \\
\bar{H}=\frac{H \alpha^{2}}{E I},
\end{gathered}
$$

where $E$ is the Young's modulus and $I$ denotes the moment of inertia.

The radios $p=\bar{A} / \bar{C}$ and $q=\bar{B} / \bar{C}$ can also be introduced; (6) can be reduced to a more contracted form:

$$
\begin{aligned}
& \frac{d^{2} \overline{M_{3}}}{d \bar{s}^{2}}-\frac{(p-q)^{2} \bar{C}+8(p+q) \bar{H}}{8 p q \bar{C}} \overline{M_{3}} \\
& +\frac{(p-q)^{2}+(p+q)}{2 p q \bar{C}} \bar{M}_{3}^{2} \\
& -\frac{4 p q-3(p+q) \bar{M}_{3}^{3}+\frac{2}{\bar{C}}\left(\frac{d \overline{M_{3}}}{d \bar{s}}\right)^{2}=0 .}{2 p q \bar{C}^{2}}=
\end{aligned}
$$

\section{Periodic Solutions}

Modeling periodical configurations in various practical problems has attracted research interests of scientists from wide spread areas, including physicists, chemists, applied mathematicians, engineers, and biologists. Periodical configurations modeled have included morphologies of calcites, silicabarium carbonate ropes, polyethylene glycol, the microstructure of rods, cables, and ribbon, general polymer helices [22], and of course those occurring in proteins and DNA [23]. The aim of this section is to find periodical solutions of (8). In terms of the phase plane, the periodic solutions correspond to the formation of closed trajectories.
Following with the line of [21], the use of Jacobi elliptic functions and the $\mathrm{KB}$ approximation scheme, namely, the elliptic KB method, was proposed. An exact solution of (8) is assumed in the form

$$
\overline{M_{3}}(s)=\frac{a \operatorname{cn}(\omega s, m)}{1+b \operatorname{cn}(\omega s, m)},
$$

where $a, b, \delta$, and $m$ are parameters to be determined. The parameter $m$ is equal to $k^{2}$ with $k$ being the elliptic modulus.

Substituting (9) into (8) and setting the coefficients of $\mathrm{cn}^{i}(\omega s, m)(i=0,1,2,3,4)$ to zero, we can easily obtain

$$
\begin{aligned}
& 2(1-m) a \omega^{2}\left[\frac{a}{\bar{C}}-b\right]=0, \\
& \frac{(p-q)^{2} \bar{C}+8(p+q) H}{8 p q \bar{C}}+\left(1+2 b^{2}-2 m-2 b^{2} m\right) \omega^{2}=0, \\
& -3 a b \frac{(p-q)^{2} \bar{C}+8(p+q) H}{8 p q \bar{C}} \\
& +a^{2}\left[\frac{(p-q)^{2}+(p+q)}{2 p q \bar{C}}+(2 m-1) \frac{2}{\bar{C}} \omega^{2}\right]=0 \\
& -3 b^{2} \frac{(p-q)^{2} \bar{C}+8(p+q) H}{8 p q \bar{C}}+a b \frac{(p-q)^{2}+(p+q)}{p q \bar{C}} \\
& -a^{2} \frac{4 p q-3(p+q)}{2 p q \bar{C}^{2}}+\left(b^{2}-2 m-2 b^{2} m\right) \omega^{2}=0, \\
& -b^{3} \frac{(p-q)^{2} \bar{C}+8(p+q) H}{8 p q \bar{C}}+a b^{2} \frac{(p-q)^{2}+(p+q)}{2 p q \bar{C}} \\
& -a^{2} b \frac{4 p q-3(p+q)}{2 p q \bar{C}^{2}}-2 b m \omega^{2}-a m \omega^{2} \frac{2}{\bar{C}}=0 .
\end{aligned}
$$

The initial condition is as follows:

$$
\begin{gathered}
\overline{M_{30}}=\overline{M_{3}}(0)=\frac{a}{1+b}, \\
{\overline{M_{3}}}^{\prime}(0)=0 .
\end{gathered}
$$

Solving the algebraic equations (10) and (11), the solutions of $a, b, m$, and $\omega$ can be straightforwardly determined. We set $a, b, m, \omega, H$, and $\overline{M_{30}}$ as the unknowns; the solutions can be 
solved by the six equations. The exact solution can be express as follows:

$$
\begin{gathered}
a=\bar{C}, \\
b=1, \\
\omega=\sqrt{\frac{3 p+3 q-4 p q}{8 p q}}, \\
m=\frac{4 p^{2}+p(25-36 q)+q(25+4 q)}{8(p(3-4 q)+3 q)}, \\
H=\left(c \left(-(p-q)^{2}+c^{2} p q\right.\right. \\
\cdot\left(-\frac{2\left(p+(p-q)^{2}+q\right)}{c^{2} p q}\right. \\
\left.\left.\left.+\frac{4 p q-3(p+q)}{2 c^{2} p q}\right)\right)\right) \\
M_{30}=\frac{\bar{C}}{2} .
\end{gathered}
$$

The following conditions hold for the existence of a periodical solution in (8):

$$
\begin{gathered}
3 p+3 q-4 p q>0 \\
\frac{4 p^{2}+p(25-36 q)+q(25+4 q)}{8(p(3-4 q)+3 q)}>1 .
\end{gathered}
$$

In this case, the exact solution can be also expressed as

$$
\overline{M_{3}}(s)=\frac{a \operatorname{dn}(\omega s, m)}{1+b \operatorname{dn}(\omega s, m)},
$$

where

$$
\begin{gathered}
\omega=\frac{\sqrt{2\left(p+(p-q)^{2}+q\right) / p q-7(4 p q-3(p+q)) / 2 p q}}{4 \sqrt{2}}, \\
m=-4(4 p q-3(p+q)) \\
\quad\left(p q \left(\frac{2\left(p+(p-q)^{2}+q\right)}{p q}\right.\right. \\
\left.\left.-\frac{7(4 p q-3(p+q))}{2 p q}\right)\right)^{-1},
\end{gathered}
$$

and $a, b$ are the same as in (12).
According to the theories of ordinary differential equations [24], significant information can be extracted from phase plane analysis. For this aim, (8) can be expressed as

$$
\begin{gathered}
\frac{d x}{d s}=f(x, y)=y, \\
\frac{d y}{d s}=g(x, y)=\frac{(p-q)^{2} \bar{C}+8(p+q) \bar{H}}{8 p q \bar{C}} x \\
-\frac{(p-q)^{2}+(p+q)}{2 p q \bar{C}} x^{2}+\frac{4 p q-3(p+q)}{2 p q \bar{C}^{2}} x^{3}-\frac{2}{\bar{C}} y^{2} .
\end{gathered}
$$

There are three critical points

$$
\begin{gathered}
x_{1}=0, \\
x_{2}=\frac{c\left(2\left(p+p^{2}+q-2 p q+q^{2}\right)-P\right)}{4(-3 q+p(-3+4 q))}, \\
x_{3}=\frac{c\left(2\left(p+p^{2}+q-2 p q+q^{2}\right)+P\right)}{4(-3 q+p(-3+4 q))},
\end{gathered}
$$

where

$$
\begin{aligned}
P & \\
= & \sqrt{2} \\
& \quad\left(2 p^{4}+8 p^{3}(2-3 q)+p^{2}\left(23-56 q+60 q^{2}\right)\right. \\
& \left.\quad+q^{2}(23+2 q(8+q))-2 p q(-23+4 q(7+3 q))\right)^{1 / 2} .
\end{aligned}
$$

It is clear that $f(x,-y)=-f(x, y)$ and $g(x,-y)=$ $g(x, y)$; thus the system (16) is reversible [21]. As discussed in [21], a closed orbit in phase space is formed by a twin trajectory, which is shown in Figure 1. In this case, the critical point $x_{2}$ is a centre; the periodical solution is around the point; Figure 2 is in accordance with the result.

Without loss of generality, we choose $p=4 / 15, q=$ $16 / 15$, and $c=1$. By calculation, (13) is held, so there is a periodical solution as shown in Figure 2. The evolution of $\overline{M_{3}}(s)$ is shown in Figure 3, which confirms the results in Figure 2.

In (12), the frequency $\omega$ and elliptic modulus $m$ depend only on the radios $p$ and $q$, not on $\bar{C}$; it is clearly evident that the radios mainly reflect the period of the solution. Sequence dependence and anisotropy of bending persistence length has been widely noticed in the base-pair steps approaches, in which relative rotation and displacement of every two segments are defined trough six parameters slide, shift, rise, tilt, roll, and twist [25]. Looking at microscopic structure of DNA, macromolecule suggests that bending toward the groove is easier than bending toward the backbone [26], which is confirmed by Monte Carlo simulations. Thus, the asymmetry may be an important factor in DNA reconfiguration process. 


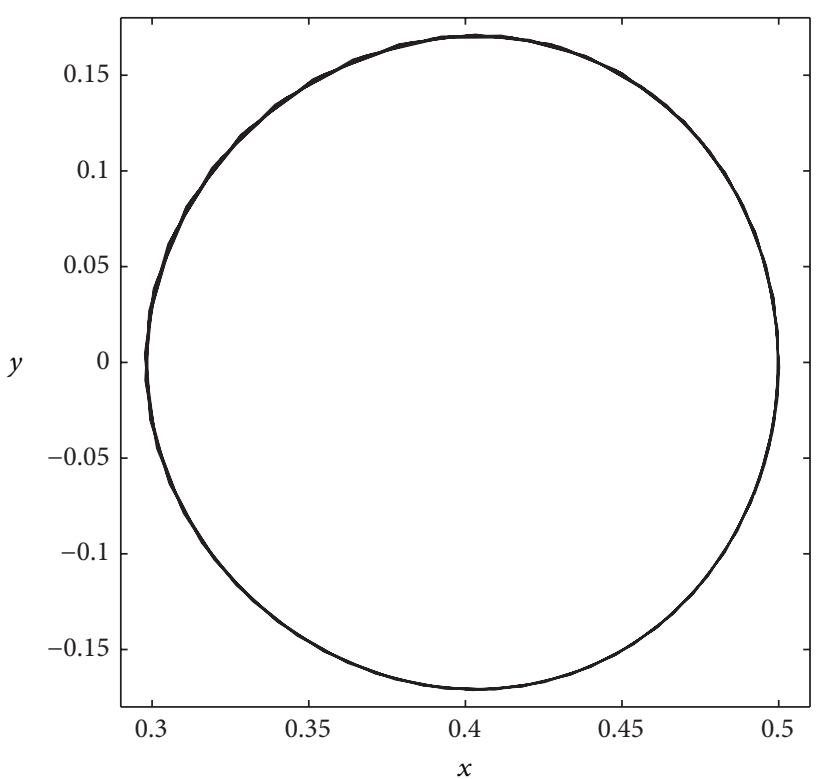

Figure 2: Phase space of (16).

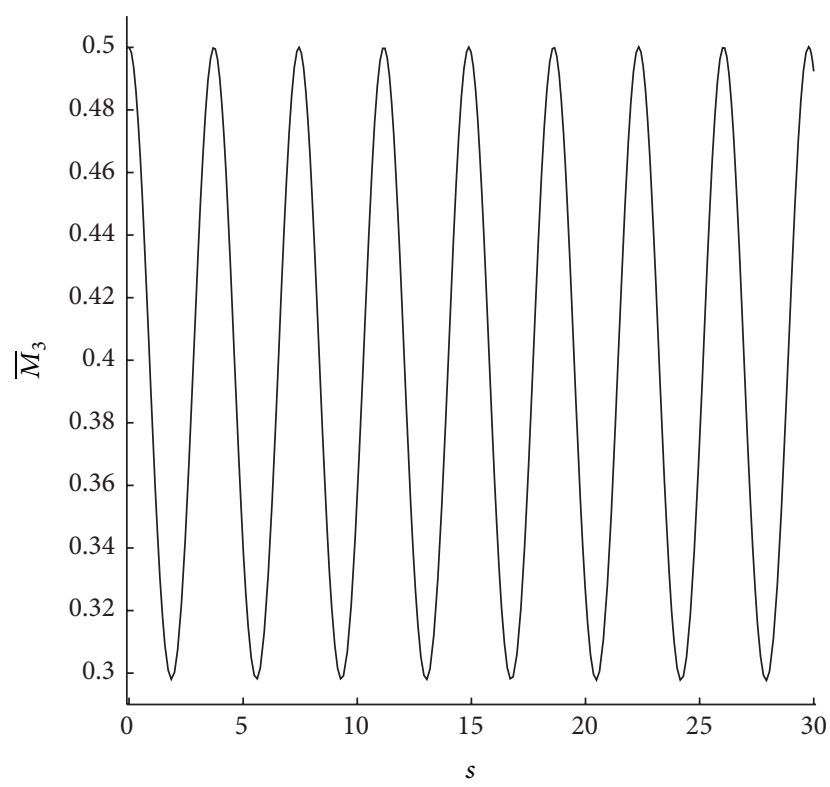

FIgURE 3: The evolution of $\overline{M_{3}}(s)$.

In the following, the simulations of DNA configurations are done under different radios between $p$ and $q$.

Experimental results show that the elastic thin rod model considered DNA molecular internal structure and external environment as a whole, which significantly shows asymmetry. As shown in [26], $q / p$ varies from 1 to 4 . The effects of the asymmetry on the frequency $\omega$ and elliptic modulus $m$ in (12) are studied in Figure 4. Figure 5 shows the period $T$ of the periodical solution changes with the asymmetry $q / p$.

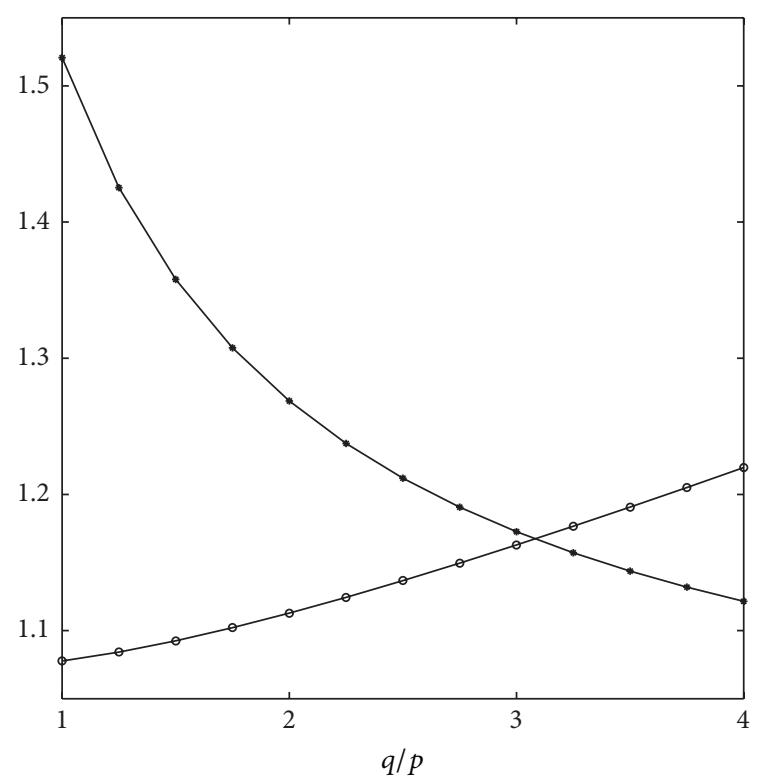

$\rightarrow \omega$

FIgURE 4: Variations of $m$ and $\omega$ with respect to $q / p$.

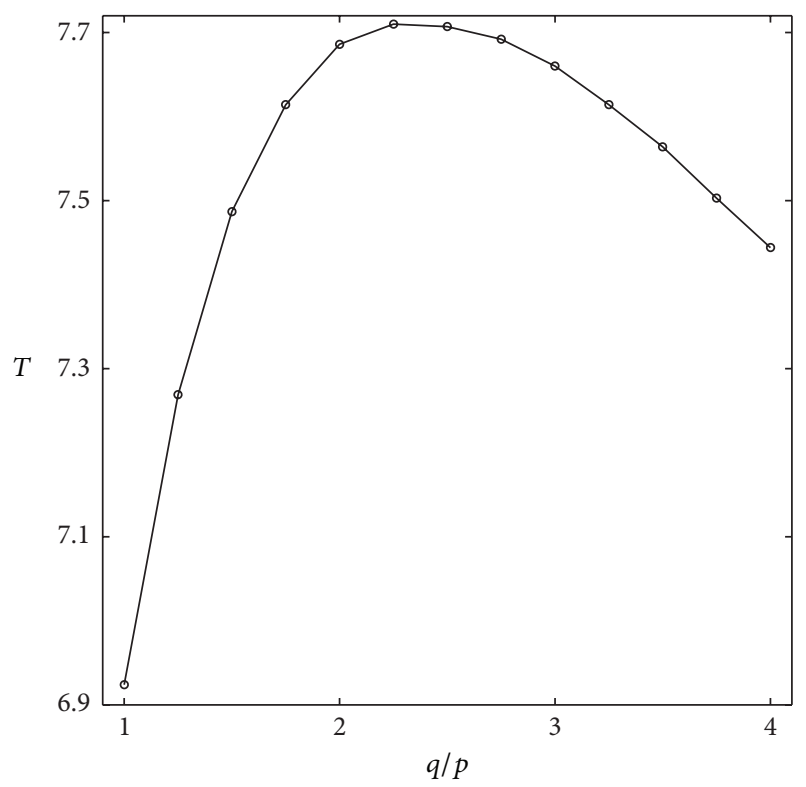

FIgURE 5: Variations of the period $T$ with respect to $q / p$.

The topological parameter, twisting number $T_{\omega}$, is defined as [12]

$$
T_{\omega}=\frac{1}{2 \pi} \int_{0}^{L} \omega_{3}(s) d s .
$$

Figure 6 shows the twisting number of the elastic rod per unit length with respect to the asymmetry $q / p$. From Figures $4-$ 6 , we can find that DNA segment will undergo a series of alteration with the change of the asymmetry, which shows a fairly well agreement with [27]. 


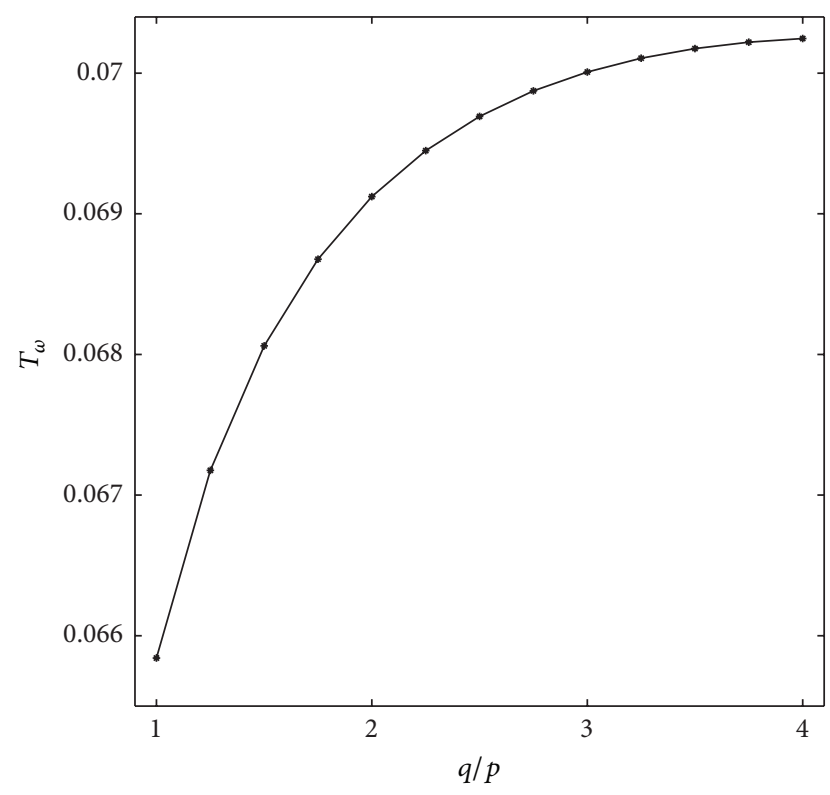

Figure 6: Variations of $T_{\omega}$ with respect to $q / p$.

\section{Conclusion}

In the present paper, we revised the reduced form of Kirchhoff equations, which characterizes the equilibrium configurations of DNA segments with the noncircular cross-section. The Jacobi elliptic Krylov-Bogoliubov (KB) method is used to find one family of exact periodical solutions of the Kirchhoff equations. The effect of the asymmetry on the equilibrium configurations of DNA is discussed; the results show that the asymmetry is an important factor in the process of DNA elastic rod reconfiguration, which shows a fairly well agreement with [27].

Finally, it should be noted that the interfacial energy model is only a coarse-grained model. Many data of DNA is still measured in future experience, such as the presence of DNA segments environment. The DNA configuration with periodically varying bending rigidities will be also investigated in future work.

\section{Conflict of Interests}

The authors declare that there is no conflict of interests regarding the publication of this paper.

\section{Acknowledgments}

This work was supported by the National Nature Science Foundation of China (no. 11372210), Research Fund for the Doctoral Program of Higher Education of China (no. 20120032110010), and the Tianjin Research Program of Application Foundation and Advanced Technology (no. 12JCZDJC28000). This support is greatly appreciated.

\section{References}

[1] K. Luger, A. W. Mäder, R. K. Richmond, D. F. Sargent, and T. J. Richmond, "Crystal structure of the nucleosome core particle at 2.8 Å resolution," Nature, vol. 389, no. 6648, pp. 251-260, 1997.

[2] S. E. Halford and J. F. Marko, "How do site-specific DNAbinding proteins find their targets?" Nucleic Acids Research, vol. 32, no. 10, pp. 3040-3052, 2004.

[3] J. Elf, G.-W. Li, and X. S. Xie, "Probing transcription factor dynamics at the single-molecule level in a living cell," Science, vol. 316, no. 5828, pp. 1191-1194, 2007.

[4] J.-I. Nishimura, Y. Murakami, and T. Kinoshita, "Paroxysmal nocturnal hemoglobinuria: an acquired genetic disease," American Journal of Hematology, vol. 62, no. 3, pp. 175-182, 1999.

[5] Z. Bryant, M. D. Stone, J. Gore, S. B. Smith, N. R. Cozzarelli, and C. Bustamante, "Structural transitions and elasticity from torque measurements on DNA," Nature, vol. 424, no. 6946, pp. 338-341, 2003.

[6] J. F. Allemand, D. Bensimon, R. Lavery, and V. Croquette, "Stretched and overwound DNA forms a Pauling-like structure with exposed bases," Proceedings of the National Academy of Sciences of the United States of America, vol. 95, no. 24, pp. 1415214157, 1998.

[7] S. B. Smith, Y. Cui, and C. Bustamante, "Overstretching B-DNA: the elastic response of individual double-stranded and singlestranded DNA molecules," Science, vol. 271, no. 5250, pp. 795799, 1996.

[8] T. Lionnet, S. Joubaud, R. Lavery, D. Bensimon, and V. Croquette, "Wringing out DNA," Physical Review Letters, vol. 96, no. 17, Article ID 178102, 4 pages, 2006.

[9] C. Bustamante, J. C. Macosko, and G. J. L. Wuite, "Grabbing the cat by the tail: manipulating molecules one by one," Nature Reviews Molecular Cell Biology, vol. 1, no. 2, pp. 130-136, 2000.

[10] T. R. Strick, V. Croquette, and D. Bensimon, "Single-molecule analysis of DNA uncoiling by a type II topoisomerase," Nature, vol. 404, no. 6780, pp. 901-904, 2000.

[11] B. Alberts, D. Bray, J. Lewis, M. Raff, K. Roberts, and J. D. Watson, Molecular Biology of the Cell, Garland Publishing, New York, NY, USA, 1994.

[12] Y. Z. Liu, Nonlinear Mechanics of Thin Elastic Rod: Theoretical Basis of Mechanical Model of DNA, Tsinghua University Press, Beijing, China, 2006.

[13] X. H. Zhou, "A one-dimensional continuous model for carbon nanotubes," The European Physical Journal B-Condensed Matter and Complex Systems, vol. 85, pp. 1-8, 2012.

[14] X. H. Zhou, "Some notes on low-dimensional elastic theories of bio- and nano-structures," Modern Physics Letters B, vol. 24, no. 23, pp. 2403-2412, 2010.

[15] Y. Shi and J. E. Hearst, "The Kirchhoff elastic rod, the nonlinear Schrödinger equation, and DNA supercoiling," The Journal of Chemical Physics, vol. 101, no. 6, pp. 5186-5199, 1994.

[16] Y. Xue, Y.-Z. Liu, and L.-Q. Chen, "The Schrödinger equation for a Kirchhoff elastic rod with noncircular cross section," Chinese Physics, vol. 13, no. 6, pp. 794-797, 2004.

[17] W. Wang, Q.-C. Zhang, and G. Jin, “The analytical reduction of the Kirchhoff thin elastic rod model with asymmetric cross section," Acta Physica Sinica, vol. 61, no. 6, Article ID 064602, 2012.

[18] W. Wang, Q.-C. Zhang, and Q.-Z. Xie, "Analytical reduction of the non-circular Kirchhoff elastic rod model with the periodically varying bending rigidities," Physica Scripta, vol. 87, no. 4, Article ID 045402, 6 pages, 2013. 
[19] A. Elías-Zúñiga and O. Martínez-Romero, "Accurate solutions of conservative nonlinear oscillators by the enhanced cubication method," Mathematical Problems in Engineering, vol. 2013, Article ID 842423, 9 pages, 2013.

[20] A. Elias-Zuniga, D. O. Trejo, I. F. Real, and O. Martinez-Romero, "A transformation method for solving conservative nonlinear two-degree-of-freedom systems," Mathematical Problems in Engineering, vol. 2014, Article ID 237234, 14 pages, 2014.

[21] S. K. Lai and K. W. Chow, "Exact solutions for oscillators with quadratic damping and mixed-parity nonlinearity," Physica Scripta, vol. 85, no. 4, Article ID 045006, 2012.

[22] M. Barros and A. Ferrández, "A conformal variational approach for helices in nature," Journal of Mathematical Physics, vol. 50, no. 10, Article ID 103529, 20 pages, 2009.

[23] M. Yavari, "B- to Z-DNA transition probed by the Feoli's formalism for a Kirchhoff model," International Journal of Modern Physics B, vol. 27, no. 23, Article ID 1350121, 2013.

[24] E. A. Coddington and N. Levinson, Theory of Ordinary Differential Equations, Tata McGraw-Hill Education, 1955.

[25] W. K. Olson, N. L. Marky, R. L. Jernigan, and V. B. Zhurkin, "Influence of fluctuations on DNA curvature: a comparison of flexible and static wedge models of intrinsically bent DNA," Journal of Molecular Biology, vol. 232, no. 2, pp. 530-554, 1993.

[26] A. Balaeff, L. Mahadevan, and K. Schulten, "Modeling DNA loops using the theory of elasticity," Physical Review E: Statistical, Nonlinear, and Soft Matter Physics, vol. 73, no. 3, Article ID 031919, 23 pages, 2006.

[27] F. Mohammad-Rafiee and R. Golestanian, "The effect of anisotropic bending elasticity on the structure of bent DNA," Journal of Physics: Condensed Matter, vol. 17, no. 14, pp. S1165S1170, 2005. 


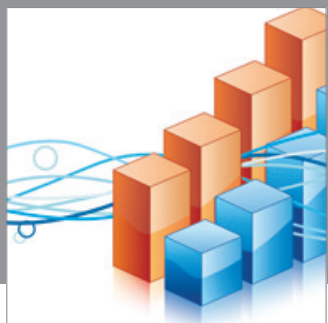

Advances in

Operations Research

mansans

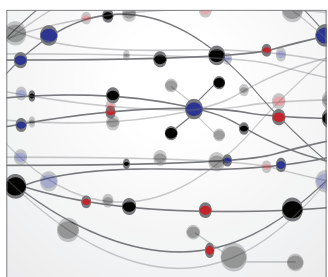

The Scientific World Journal
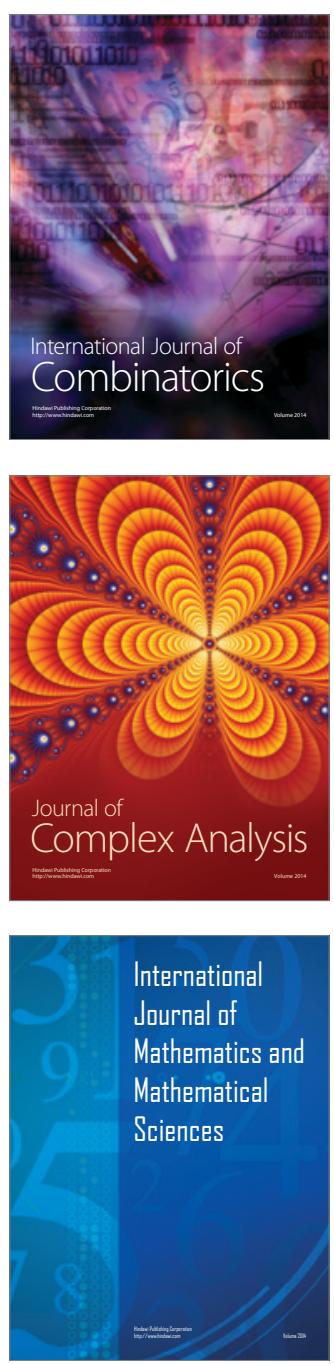
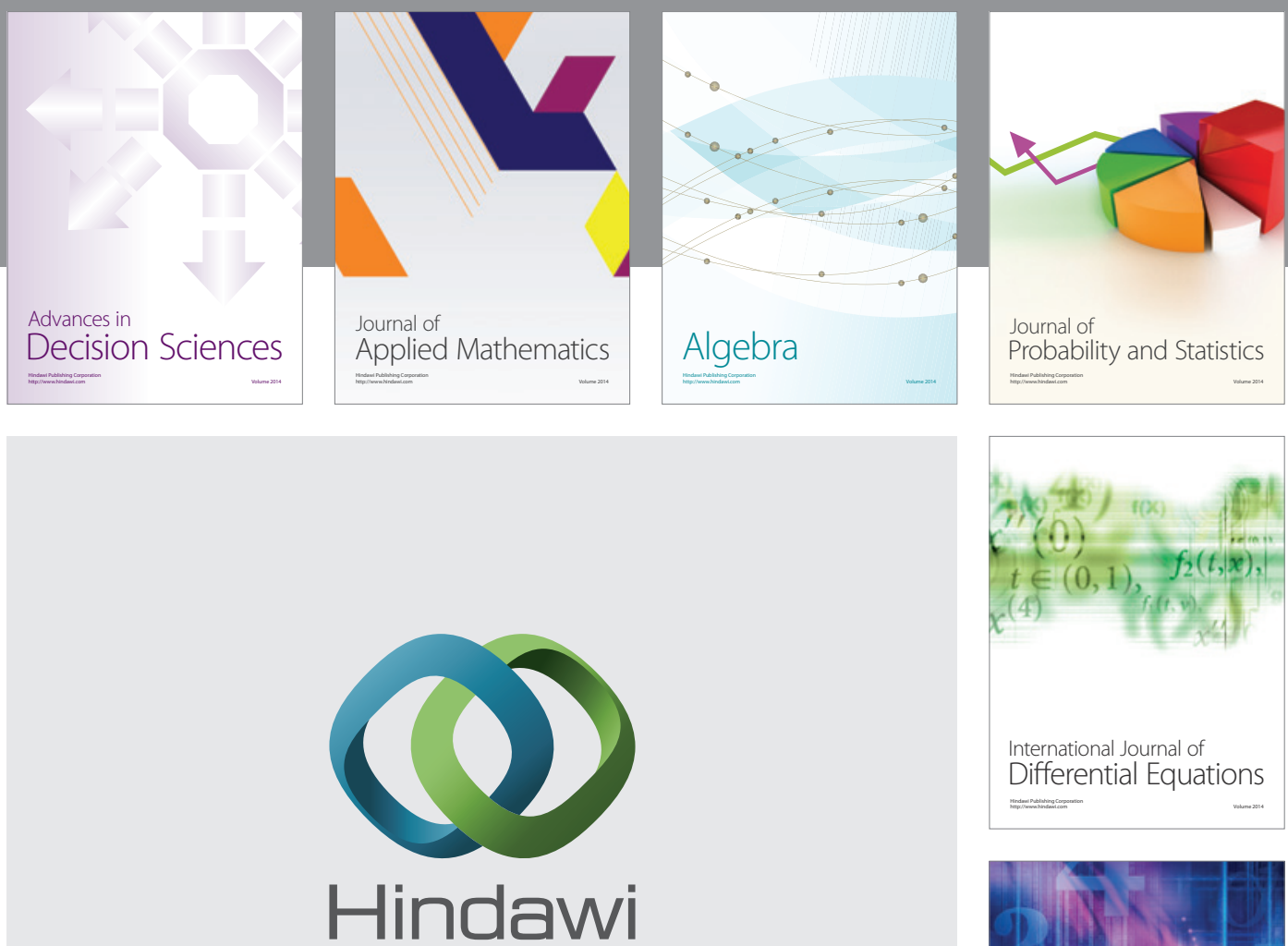

Submit your manuscripts at http://www.hindawi.com
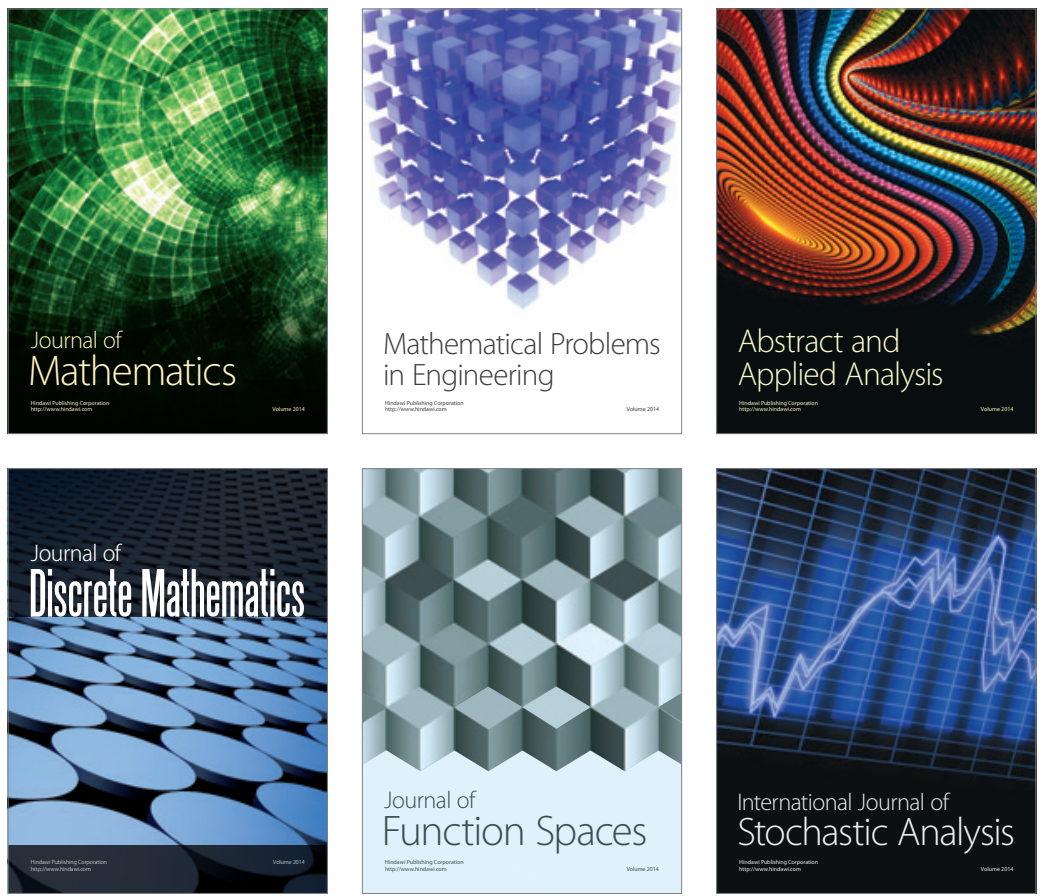

Journal of

Function Spaces

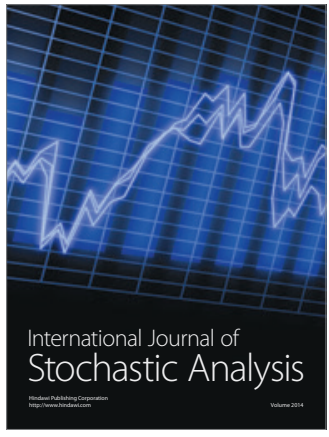

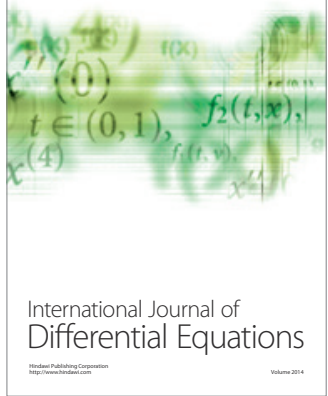
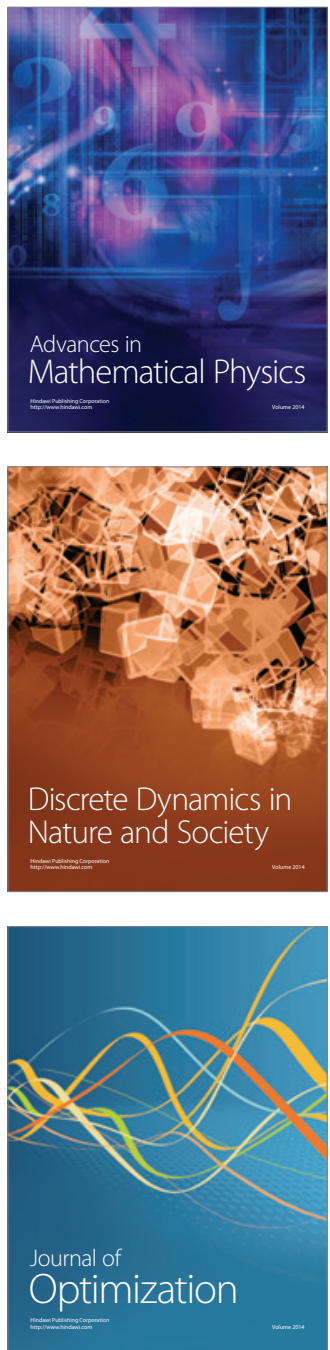